\title{
Parametric Estimation of NMR Spectroscopy Using Matrix Pencil
}

\author{
Javad Razavilar, Y. Li and K. J. Ray Liu \\ Electrical Engineering Department \\ University of Maryland \\ College Park, MD 20742 \\ Fax:1-301-405-6707 \\ Email: javad(liye, kjrliu)@src.umd.edu
}

\begin{abstract}
Abstrect - To determine protein structure by means of NMR spectroscopy, the parameters of NMR spectroscopy have to be estimated. This paper proposes a novel parameter estimation algorithm for NMR spectroscopy. The new algorithm is based on the matrix pencil consisting of the NMR data. Compared with the Kumaresan-Tufts (KT) algorithm [1], The new algorithm improves the noise threshold by about $6 \mathrm{~dB}$. Its effectiveness is demonstrated by computer simulation.
\end{abstract}

Key words: NMR spectroscopy, damped sinusoid, and matrix pencil

\section{INTRODUCTION}

To determine protein structure by means of NMR spectroscopy, the parameters of NMR spectroscopy have to be estimated. At early stages of NMR spectroscopy analysis, the application of discrete Fourier transform (DFT) has made a great progress in this field. However, the frequency resolution of the DFT-based algorithms is limited by the short aquisition time of the NMR signal and measurement noise. Since NMR spectroscopy can be modeled as the summation of damped sinusoids, the model-based algorithms have been used in the parameter estimation of NMR spectroscopy to improve the frequency resolution. The Kumaresan-Tufts (KT) algorithm [1] is one of the most effective algorithms. But, it is still sensitive to measurement noise. Therefore, it has high noise threshold. To alleviate noise effect and improve resolution, we propose a matrix pencil algorithm in this paper.

\section{MATHEMATICAL MODEL OF NMR SPECTROSCOPY}

Before developing our algorithm, we will briefly describe the mathematical model of NMR spectroscopy. The detailed description is given by [2].

Discrete NMR spectroscopy can be expressed as [2]

$$
x(n)=\sum_{k=1}^{K} c_{k} e^{2 k n}
$$

where $K$ is the order of the signal, $s_{k}=-\alpha_{k}+\omega_{k}$, and $\omega_{k} \in[-\pi, \pi], \alpha_{k} \in \mathcal{R}^{+}$, which is called the damping factor. The larger the $\alpha_{k}$, the faster the sinusoid with frequency

The work was supported in part by the NIH grant 1R01GM49707 and the NSF grants MIP9309506 and MIP9457397. $\omega_{k}$ decays. Without loss of generality, we suppose that $s_{k}$ be distinct. If the measurement error or noise $\{w(n)\}$ is considered, the measured NMR data can be expressed as

$$
y(n)=x(n)+w(n),
$$

for $n=0,1, \cdots, N-1$. Normally, we have to make sure $N \geq 2 K$ to estimate the frequencies and the damping factors of NMR spectroscopy.

\section{Algorithm DeVElopMENT}

From the measured data, we first set $K \times K$ data matrices

$$
\mathbf{A}_{n}=\left(\begin{array}{lll}
y(n) & \cdots & y(n+K-1) \\
y(n+1) & \cdots & y(n+K) \\
\vdots & \vdots & \vdots \\
y(n+K-1) & \cdots & y(n+2 K-2)
\end{array}\right)
$$

for $n=0,1, \cdots, N-2 K+1$. From (1) and (2), it can be shown that

$$
A_{n}=S^{T} \mathbf{C} \Phi^{n} \mathbf{S}+W_{n},
$$

where

$$
\mathbf{W}_{n}=\left(\begin{array}{lll}
w(n) & \cdots & w(n+K-1) \\
w(n+1) & \cdots & w(n+K) \\
\vdots & \vdots & \vdots \\
w(n+K-1) & \cdots & w(n+2 K-2)
\end{array}\right)
$$

In the above expression,

$$
\begin{gathered}
\operatorname{diag}\{\Phi\}=\left[e^{s_{1}}, e^{s_{2}}, \cdots, e^{s_{K}}\right], \\
\operatorname{diag}\{\mathbf{C}\}=\left[c_{1}, c_{2}, \cdots, c_{K}\right] \\
\mathbf{S}=\left[\dot{\mathbf{r}}\left(s_{1}\right), \mathbf{r}\left(s_{2}\right), \cdots, \mathbf{r}\left(s_{K}\right)\right]^{T},
\end{gathered}
$$

where

$$
\mathbf{r}\left(s_{k}\right)=\left[1, e^{s_{k}}, \cdots, e^{(K-1) s_{k}}\right]^{T} .
$$

If there is no measurement noise, then $\mathbf{W}_{n}=0$ for all $n=0,1, \cdots, N-2 K+1$. Since $s_{k}$ for $k=1,2, \cdots, K$ are distinct, $S$ and $\Phi$ are of full rank. Hence, $A_{n}$ for $n=0,1, \cdots, N-2 K+1$ are invertable and

$$
A_{n}^{-1} A_{n+1}=S^{-1} \Phi S \text {. }
$$

Therefore, the eigrnvalues of $A_{n}^{-1} A_{n+1}$ are $e^{s_{1}}, e^{s_{2}}, \cdots, e^{s_{K}}$ if there is no measurement noise. Hence, $s_{k}$ can be estimated from the eigenvalues of $\mathbf{A}_{n}^{-1} \mathbf{A}_{n+1}$. 


\section{Selection of Weighing Factors}

The estimation performance of $s_{k}$ relys on how accurate the estimation of $\mathbf{S}^{-1} \boldsymbol{\Phi} \mathbf{S}$ is. To obtain a good estimation of $P=S^{-1} \Phi S$, we construct the following statistics

$$
\widehat{\mathbf{P}}=\sum_{n=0}^{N-2 K} a_{n} \mathbf{A}_{n}^{-1} \mathbf{A}_{n+1},
$$

where $a_{n}$ 's are the weighing factors to be determined. To make the estimation unbiased, we must choose $\sum_{n=0}^{N-2 K} a_{n}=1$.

From (4), if the signal-to-noise ratio is high, by direct matrix calculation, we have

$$
\widehat{\mathbf{P}} \approx \mathrm{P}+\sum_{n=0}^{N-2 K} a_{n} \overline{\mathrm{W}}_{n},
$$

where $\bar{W}_{n}$ represents the effect of the measurement noise which can be written as

$$
\bar{W}_{n}=A_{n}^{-1}\left\{W_{n} P+W_{n+1}\right\} \text {. }
$$

From the statistics theory, to minimize $\|\widehat{\mathbf{P}}-\mathbf{P}\|, a_{n}$ should be inverse proprotional to the variance of the elements in $\bar{W}_{n}$. Hence,

$$
a_{n} \propto \frac{\left|\operatorname{det}\left(\mathbf{A}_{n}\right)\right|^{2 / K}}{\operatorname{Var}(w(n))} .
$$

To reduce the effect of the measurement noise, the rankdeficient and Hankel properties of the prediction matrix can be used to supress noise in advance as described in [3]. After the noise reduction,

$$
\operatorname{Var}\left(w_{n}\right) \propto L+1-|L-n|, \quad L=\lceil N / 2\rceil .
$$

Therefore, the optimum $a_{n}$ will be

$$
a_{n}=\frac{\left|\operatorname{det}\left(\mathbf{A}_{n}\right)\right|^{2 / K}(L+1-|L-n|)}{\sum_{k}\left|\operatorname{det}\left(\mathbf{A}_{k}\right)\right|^{2 / K}(L+1-|L-k|)} .
$$

As such, from (11) and (16), we can get the optimum estimation of $P$. By means of it, the parameters of the NMR signal can be estimated.

\section{COMPUTER Simulation}

We will test the performance of the new algorithm and compare it with the KT algorithm [1] by a computer simulation example.

The simulated data are generated by

$$
y(n)=e^{s_{1} n}+e^{s_{2} n}+w(n), \quad n=0,1, \cdots, 24
$$

where $s_{1}=-0.2+\jmath 2 \pi(0.42), s_{2}=-0.1+\jmath 2 \pi(0.52)$, and $w(n)$ is complex white Gaussian noise with variance $\sigma^{2}$. The SNR used in this paper is the peak signal-to-noise ratio defined as

$$
\operatorname{SNR}=10 \log \left(\frac{1}{2 \sigma^{2}}\right)
$$

The MSE's of $\alpha_{1}, \omega_{1}, \alpha_{2}$ and $\omega_{2}$ for the new algorithm and the KT algorithm are shown in Figure 1 (a)-(d). From these figures, the noise threshold of our new algorithm is about $5 d B$ lower than that of KT algorithm.

\section{Conclusion}

This paper propose a novel parameter estimation algorithm for NMR spectroscopy. Computer simulation demonstrates that the new algorithm can estimate the parameter of NMR spectroscopy effectively. We are currently trying to generalize the algorithm to the parameter estimation of multi-dimensional NMR spectroscopy.

\section{REFERENCES}

[1] R. Kumaresan and R. W.Tufts, "Estimation the parameters of exponentially damped sinusoids and pole-zero modelling in noise," IEEE Trans. on Acoust., Speech, Signal Processing, vol. 30, pp. 833-840, Dec. 1982.

[2] A. Bax, "A simple description of two-dimensional NMR spectroscopy," Bulletin of Magnetic Resonance vol.7, pp. 167-183, 1985.

[3] Y. Li, K. J, R. Liu, J. Razavilar, "Improved parameter estimation schemes for damped sinusoidal signals," Technical Report of SRC of University of Maryland, T.R. 95-11, 1995.

[4] S. Haykin, Adoptive Filter Theory, Prentice Hall, 1991.
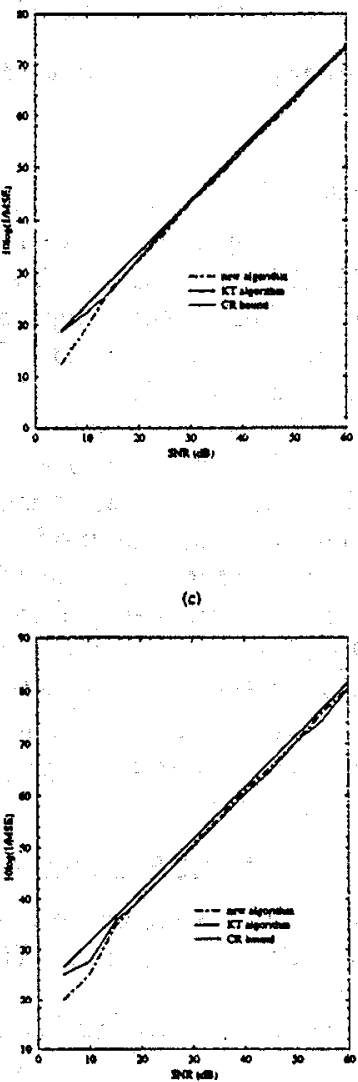

(b)

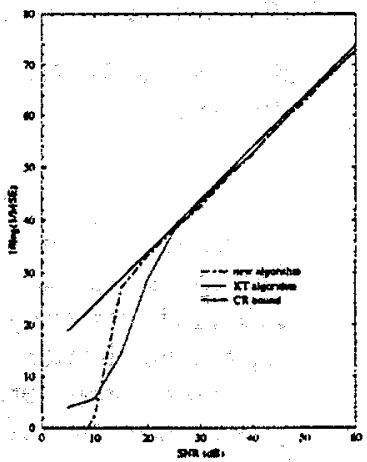

(d)

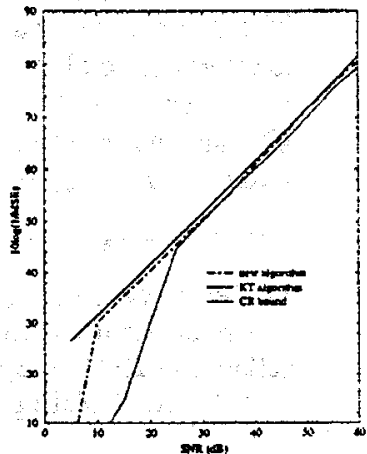

Fig. 1. The MSE of (a) $\alpha_{1}$, (b) $\omega_{1}$, (c) $\alpha_{2}$ and (d) $\omega_{2}$ obtained in 500 trails of the new algorithm and $\mathrm{KT}$ algorithm when $s_{1}=-0.2+\jmath 2 \pi .0 .42, s_{2}=-0.1+\jmath 2 \pi 0.52$ and $N=25$. 